\title{
Proceeding
}

Supplementary Issue: Spring Conferences of Sports Science. Costa Blanca Sports Science Events, 14-15 June 2019. Alicante, Spain.

\section{Examining effectiveness of e-sports activity in Japan}

\author{
GOICHI HAGIWARA 19 , DAISUKE AKIYAMA ${ }^{2}$, SHUNICHI TAKESHITA ${ }^{1}$
}

${ }^{1}$ Department of Sports Humanities and Applied Social Science, Faculty of Physical Education, National Institute of Fitness and Sports in Kanoya, Kanoya-shi, Kagoshima, Japan

${ }^{2}$ Department of Sport Science and Health, Faculty of Human Science, Kyushu Sangyo University, Fukuoka, Japan

\begin{abstract}
Digital games or video games have a positive impact on cognitive function. However, there is no study examining the positive effects of e-sports in Japan. Thus, we conducted two studies. Study 1 examined the relationship between e-sports and cognitive function using the Stroop and reverse-Stroop Color and Word Test. Additionally, study 2 examined the psychological statement while playing e-sports using biological evaluation. Study 1 included six male collegiate students who could play e-sports (three in the e-sports group, three in the control group), while study 2 involved two male Japanese collegiate e-sports players. The esports group played e-sports for 10 minutes and their cognitive function and psychological statement were evaluated during play. EEG was employed to evaluate the psychological statement of the e-sport players in study 2. Emotional condition (concentration) was analysed from the combination of the extracted raw EEG data using KANSEI Module Logger. Results of study 1 indicated that 10 minutes of e-sports activity significantly improved cognitive function, especially interference and concentration, in the e-sports group. Study 2 concluded that the average concentration value at play time was significantly higher than that during the resting period. Therefore, this study indicated that e-sports activity might have positive effects when played for a brief time. Keywords: EEG; Cognitive function; KANSEI.
\end{abstract}

\section{Cite this article as:}

Hagiwara, G., Akiyama, D., \& Takeshita, S. (2019). Examining effectiveness of e-sports activity in Japan. Journal of Human Sport and Exercise, 14(4proc), S1038-S1045. doi:https://doi.org/10.14198/ihse.2019.14.Proc4.66

Corresponding author. Department of Sports Humanities and Applied Social Science, Faculty of Physical Education, National Institute of Fitness and Sports in Kanoya, Kanoya-shi, Kagoshima, Japan.

E-mail: hagiwara-g@nifs-k.ac.jp

Supplementary Issue: Spring Conferences of Sports Science. Costa Blanca Sports Science Events, 14-15 June 2019. Alicante, Spain.

JOURNAL OF HUMAN SPORT \& EXERCISE ISSN 1988-5202

(c) Faculty of Education. University of Alicante

doi:10.14198/jhse.2019.14.Proc4.66

S1038 | 2019| Proc4 | VOLUME 14

C 2019 University of Alicante 


\section{INTRODUCTION}

The e-sports generally refers to entertainment, competition, and sports using electronic devices and is the competition between players using digital games or video games as sports competitions (Kakei, 2017). Recently, e-sports was accepted as a sports activity. However, a marketing research in Japan indicated that a large number of Japanese people view e-sports as a game and not a sport, as many thinks that sports implies "moving the body" (Cross Marketing, 2018).

Incidentally, several studies have mentioned that digital games or video games have a positive impact on cognitive function (Green, et al., 2003; Pessoa, 2009; Anguera, et al., 2013). However, there is no study examining the positive effects of e-sports in Japan. Therefore, we conducted two studies. The first one examined the relationships between e-sports activity and cognitive function using the Stroop Color and Word Test (SCWT). Studies about cognitive function have employed SCWT and have indicated the relationship between test performance and cognitive function (Song \& Hakoda, 2015). Thus, here the SCWT was adopted to measure how selective attention and cognitive flexibility were affected by e-sports activity.

Study 2 examined psychological statements while playing e-sports by using biological evaluation. The electrical activity in the brain is often used as an objective evaluation index that employs biological data. Brainwaves are generally classified into four types according to their frequency range $(0.5-4 \mathrm{~Hz}$ : delta waves; 4-8 Hz: theta waves; 8-13 Hz: alpha waves; 13-40 Hz: beta waves), and emotions can be associated with each type (Okubo, 2018) (Table. 1). During human activity, a frequency exceeding $4 \mathrm{~Hz}$ appears. To measure emotions during human activity, therefore, alpha and beta waves are used. Hence, human emotions are measured using frequency wave bands. It has been suggested, however, that judging emotions using only frequency wave bands, as described above, is far too speculative, and that the method cannot reveal a person's emotions adequately (Mitsukura, 2016). Methods are therefore being proposed for assessing emotions by segmenting the frequency wave bands (Shibata et al., 2015), and using a combination of the appearance ratios of a particular frequency wave band (Okubo, 2018; Mitsukura, 2016). Mitsukura (2016) defined emotions such as stress, concentration, drowsiness, interest, and likes/dislikes using a combination of frequencies appearing in electroencephalograms (EEGs), showing that it can be utilized as an index for evaluating emotions. Nideffer (1985) indicated that concentration is the key for successful sport performance, and Gould et al. (1992) found that higher level performance athletes are more likely to maintain higher levels of concentration than low performing athletes. These findings indicated that degree of concentration might be associated with athletic performance. Therefore, this study adopted concentration as a psychological statement while playing e-sports.

Table 1. Type of brain wave

\begin{tabular}{|c|c|c|}
\hline Type of brain wave & Frequency $(\mathrm{Hz})$ & Psychological status \\
\hline Delta wave & $0.5 \sim 4 \mathrm{~Hz}$ & non-REM sleep, unconscious \\
\hline Theta wave & $4 \sim 8 \mathrm{~Hz}$ & Sleep onset, illusion \\
\hline Alpha wave & $8 \sim 13 \mathrm{~Hz}$ & Relax \\
\hline Beta wave & $13 \sim 40 \mathrm{~Hz}$ & Arousal \\
\hline
\end{tabular}




\section{METHODS}

\section{Participants}

In study 1, the participants included six male collegiate students who could play e-sports (three in the esports group, three in the control group), and study 2 included two male Japanese collegiate e-sports players. The research team informed the participants of the purpose of the study and gave instructions on the experiment prior to participation. Data and informed consent were obtained from the participants. Participation was entirely voluntary.

\section{Procedure}

Pro Evolution Soccer 2019 (Konami, 2018) (soccer game) was adopted as an e-sports program. In Study 1, all participants took an SCWT before the e-sports group played e-sports for 10 minutes. Meanwhile the control group remained at rest. After 10 minutes, all participants took a post SCWT. In study 2, an EEG was employed to evaluate psychological statement of e-sports players. Concentration was analysed from the combination of the extracted raw EEG data by using KANSEI Module Logger (Littlesoftware, 2018).

\section{Evaluating psychological statement}

Evaluations of cognitive function

The SCWT has been widely used as a neuropsychological test to measure selective attention, cognitive function, processing speed, and interference control (Song \& Hakoda, 2011). Thus, this study adopted the Stroop/reverse-Stroop Test (Hakoda \& Sasaki, 1990, 1991) to evaluate the level of interference control. The test was composed of four subtests in which all color-word combinations and color patches were printed on four separate sheets of paper (details in Hakoda \& Sasaki, 1990, 1991). In this study, we calculated the number of achieved tasks and reverse-Stroop interference rate for each subtest.

\section{Evaluations of biological concentration while playing e-sports}

This study adopted a simple band-type EEG that only measures the Fp1 point as defined by the international 10-20 system. Since Fp1 is located on the left frontal lobe, the possible noise interference due to hair was no cause for worry; and the EEG obtained from Fp1 has been shown to be suitable for obtaining data on people's psychological condition (Mitsukura, 2016; Hotta et al., 2017). We obtained data on the intensity of alpha and beta waves at one-second intervals by having the participants wear an EEG recorder on their heads, and attaching the electrodes to their ears and foreheads. Intensity was calculated by subjecting the EEG's raw data to Fourier transform. The data thus obtained were recorded in a smartphone, and a KANSEI Module Logger (Littlesoftware Inc., 2018) that can output the data as sensitivity values was used. This study used concentration as an index. In converting the sensitivity values, we used the obtained data as the basis for cutting out wave shapes from the frequency wave bands ranging from $0.5 \mathrm{~Hz}$ to $40 \mathrm{~Hz}$, and analysed them using an algorithm. Here, regarding concentration, we used data that had been extracted from alpha waves $(8-13 \mathrm{~Hz})$ and beta waves $(13-40 \mathrm{~Hz})$, which are brain wave frequency bands that become prominent during human activity. We define concentration by calculating the occurrence ratio, especially from beta waves, which are produced when a person is in an alert state. Concentration is shown in numbers ranging from $0-100$, with higher numerical values representing higher degree of concentration.

\section{Analysis}

Study 1 used t-test to compare the number of tasks achieved before and after the experiment in the e-sports and control groups, and two-way ANOVA was adopted to compare reserve-Stroop interference rate in the esports and control groups. Study 2 used t-test to compare the degree of concentration when playing e-sports and at rest. IBM SPSS Statistics 24.0 software was used for the analysis. 


\section{RESULTS AND DISCUSSION}

Table 2 shows the number of tasks achieved for each subtests and reserve-Stroop interference rate before and after the experiment in the e-sports and control groups. In addition, the results of comparing the number of tasks achieved before and after the experiment, in the e-sports and control groups by t-test, indicated that there were significant differences in tasks 1 and 2 for the control group (Figure. 1), and tasks 2 and 3 for the e-sports group. Further, task 4 showed a marginally significant (Figure. 2).

Table 2. The number of tasks achieved for each subtests and reserve-Stroop interference rate

\begin{tabular}{|c|c|c|c|c|c|}
\hline & & \multicolumn{2}{|c|}{ pre } & \multicolumn{2}{|c|}{ post } \\
\hline & & control & e-sport & control & e-sport \\
\hline \multirow{2}{*}{ task 1} & Achieves & 75.00 & 68.33 & 86.00 & 71.00 \\
\hline & S.D. & 5.00 & 5.03 & 8.18 & 6.08 \\
\hline \multirow{2}{*}{ task 2} & Achieves & 65.67 & 54.67 & 73.00 & 61.33 \\
\hline & S.D. & 10.01 & 6.50 & 12.12 & 5.13 \\
\hline \multirow{2}{*}{ task 3} & Achieves & 56.00 & 44.00 & 58.67 & 51.33 \\
\hline & S.D. & 8.71 & 4.35 & 6.02 & 4.61 \\
\hline \multirow{2}{*}{ task 4} & Achieves & 53.67 & 39.00 & 55.33 & 44.33 \\
\hline & S.D. & 1.15 & 5.29 & 5.50 & 9.29 \\
\hline \multirow{2}{*}{$\begin{array}{l}\text { reserve-Stroop } \\
\text { interference rate }\end{array}$} & $\begin{array}{l}\text { interference } \\
\text { rate }\end{array}$ & 12.77 & 20.18 & 15.49 & 13.59 \\
\hline & S.D. & 7.51 & 3.68 & 6.02 & 1.64 \\
\hline
\end{tabular}

Number of achieving tasks for each task in control group

(pre-post)

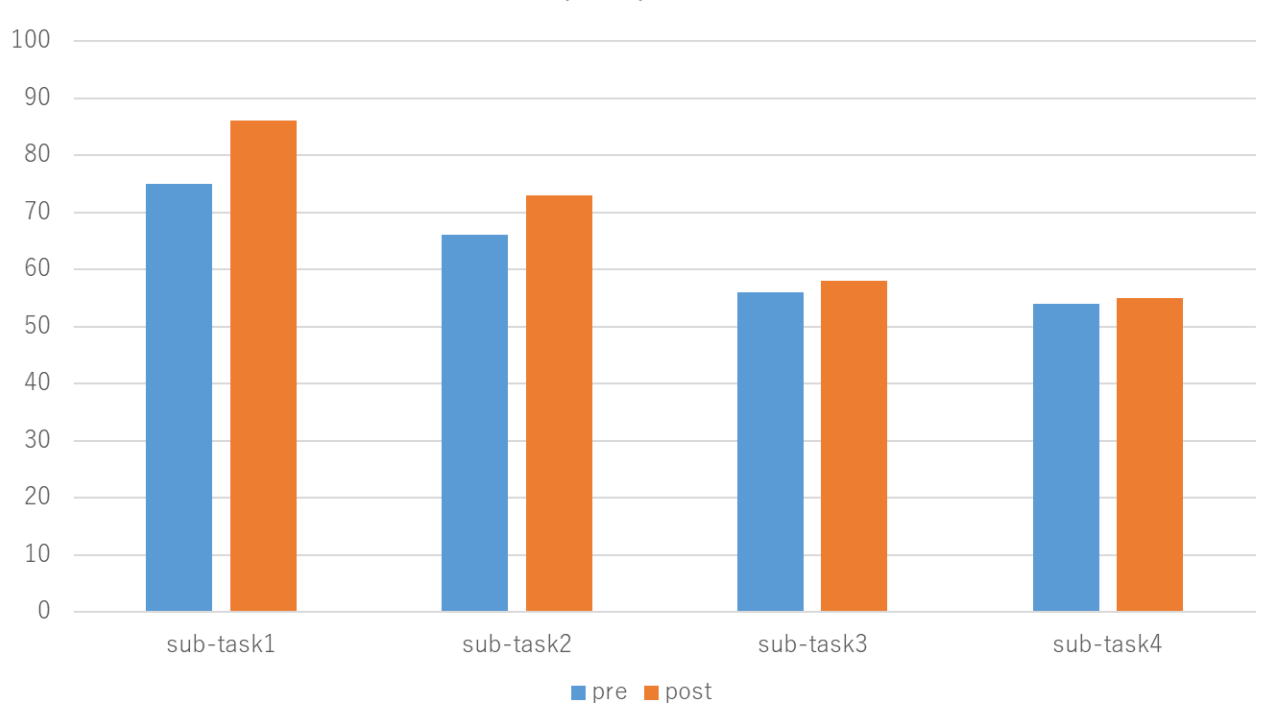

Figure 1. Results of t-test in control group. 
Number of achieving tasks for each task in e-sport group

(pre-post)

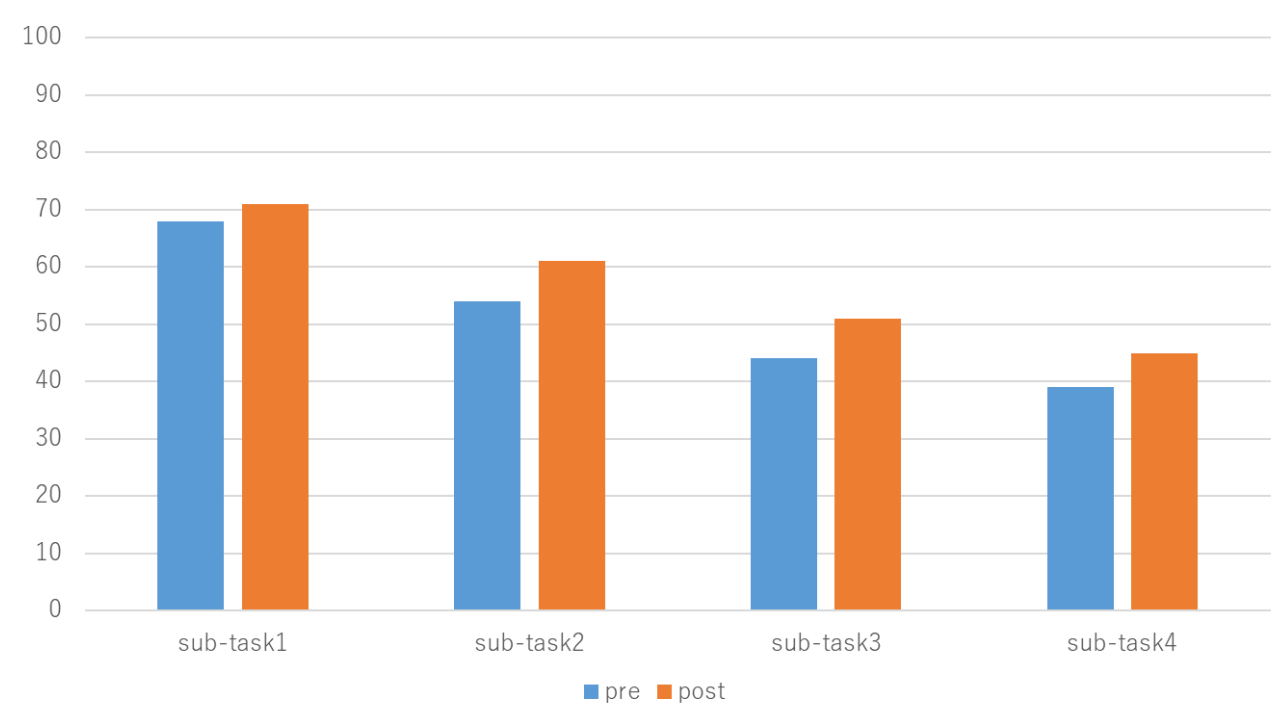

Figure 2. Results of t-test in e-sports group.

The control group showed significant improvements for the number of achievements on Task 1 and 2 but not for Task 3 and 4 . The e-sports group also showed improvement in the number of achievement for Task 2, 3, and 4 . Hence it is noted that for the e-sports group, the number of tasks achieved tends to improve towards the end of the test.

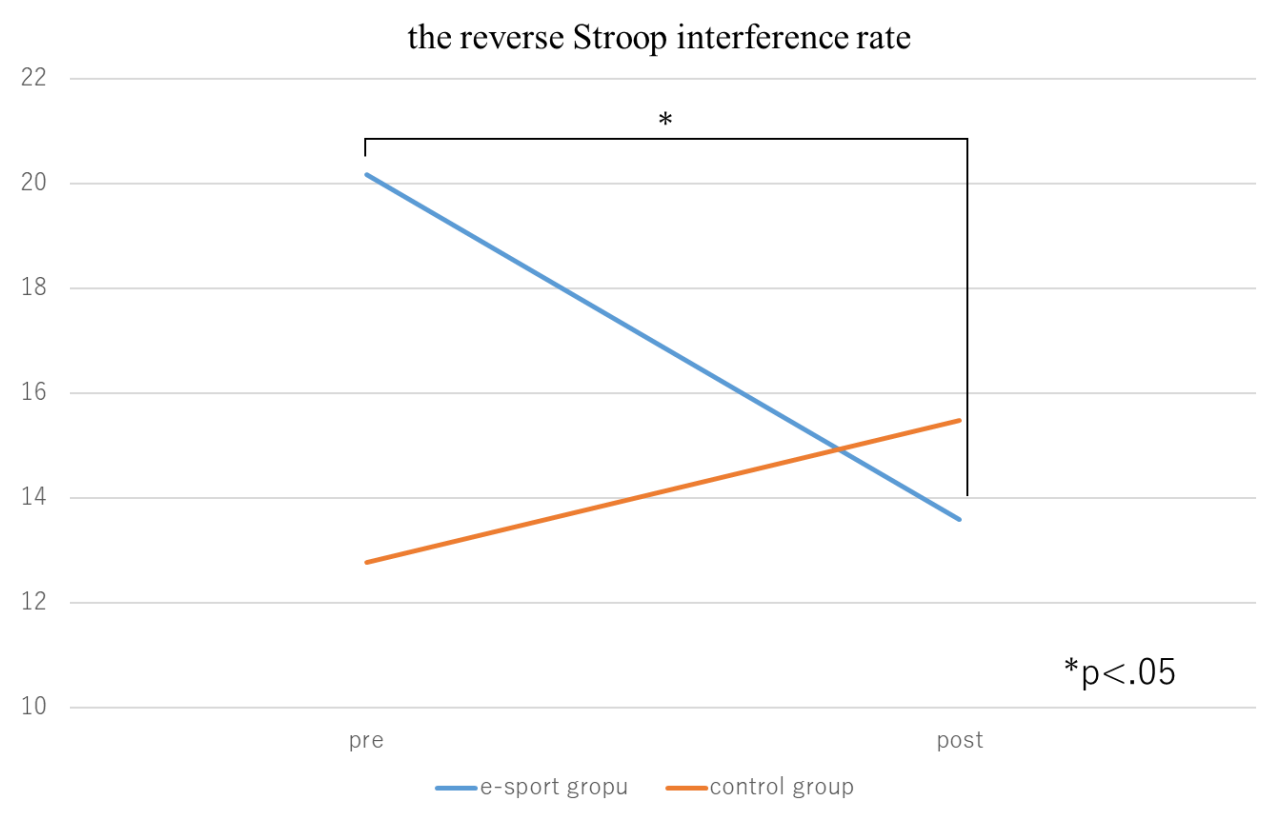

Figure 3. Result of two-way ANOVA. 
The result of the two-way ANOVA to compare reserve-Stroop interference rate in the e-sports and control groups indicated that there was no main effect of the activity condition $(F[1,4]=2.82$, ns), and the interaction between the activity condition and the activity before and after it was significant $(F[1,4]=16.26, p<.05)$. In addition, no main effects were observed before and after the activity $(F[1,4]=.45$, n.s.). Since the interaction was significant, when sub-testing the activity condition, the reverse Stroop interference rate before and after activity was not significantly different among the control and e-sports groups ( $F[1,4]=2.35$, n.S., $F[1,4]=$ .28 , n.s.). Next, we performed a subtest before and after the activity. The control group showed no significant difference in the reverse Stroop interference rate before and after the activity $(F[1,4]=2.77$, ns), but the esports group showed significant reduction $(F[1,4]=16.31, p<.05)$ (Figure. 3 ).

The result of t-test indicated that the score of concentration while playing e-sports was significantly higher than that in the resting condition $(t=12.54, p<.05)$ (Figure.4).

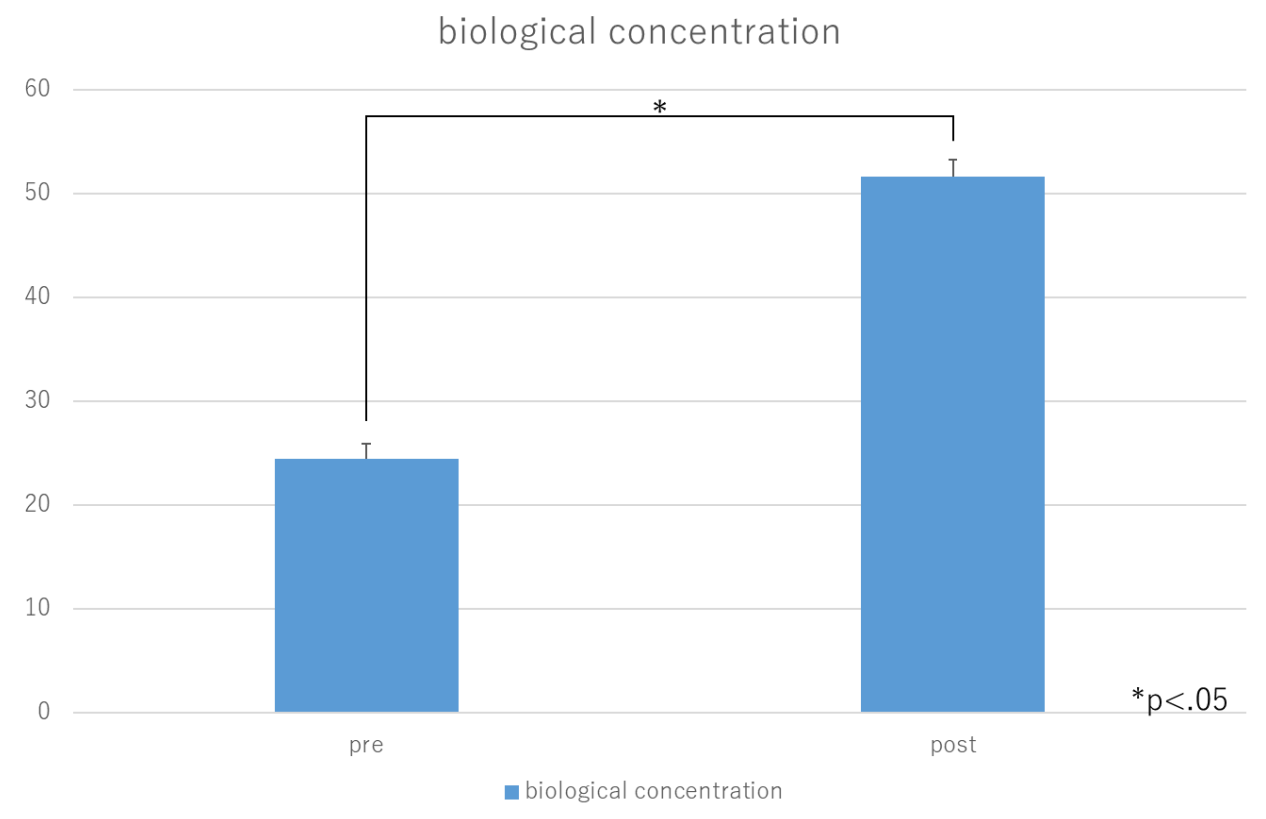

Figure 4. Result of t-test about biological concentration.

As described above, it can be seen that the reverse Stroop interference rate of the e-sports group is significantly reduced, and has changed to a state where the external environment interferes. EEG data confirmed that the concentration during e-sports tended to be significantly higher than that at rest. Thus, concentration during e-sports tends to be higher than usual. Playing e-sports might enhance concentration and players might be less likely to be influenced by other interference. Anguera et al. (2013) examined the relationship between EEG and playing digital games, demonstrating that the power of Fm $\theta$ (brain wave said to reflect attention and concentration) increased after play. Result of this study followed their result and obtained similar findings.

Several studies in sports science have been conducted on the influence of moderate physical movement on cognitive function (Byun et al., 2014; Omori, 2017). Byun et al. (2014) examined the relationship between light exercise and cognitive function and found that a person performing 10 minutes of exercise may activate a part of the brain that controls cognitive function and improves task performance. Those results suggested 
that performing appropriate physical exercise can improve cognitive function, and this study might suggest that appropriate e-sports activity can improve cognitive function.

\section{CONCLUSION}

We aimed to examine the positive effects of e-sports activity in Japan, and conducted two studies for the same. The first study examined the relationships between e-sports activity and cognitive function using Stroop and reverse-Stroop Color-Word Test. The results indicated that the e-sports group significantly improved their attention and cognitive functions after playing e-sports for 10 minutes. In addition, study 2 examined biological concentration while playing e-sports by using EEG, concluding that the average value of concentration at play time was significantly higher than that during the resting period. Therefore, this study indicated that e-sports might have positive effects when played for a brief time.

\section{REFERENCES}

Anguera, J.A., Boccanfuso, J., Rintoul, J.L., Al-Hashimi, O., Faraji, F., Janowich, J., Kong, E., Larraburo, Y., Rolle, C., Johnston, E., \& Gazzaley, A. (2013). Video game training enhances cognitive control in older adults. Nature, 501, 97-101. https://doi.org/10.1038/nature12486

Byun, K., Hyodo, K., Suwabe, K., Ochi, G., Sakairi, Y., Kato, M., Dan, I., \& Soya, H. (2014). Positive effect of acute mild exercise on executive function via arousal-related prefrontal activations: an fNIRS study. Neurolmage, 98, 336-345. https://doi.org/10.1016/j.neuroimage.2014.04.067

Cross Marketing (2018, May 31). Survey on e-sports. Retrieved from https://www.crossm.co.jp/report/sports/es20180531/

Gould, D., Eklund, R., \& Jackson, S. (1992). 1988 U.S. Olympic wrestling excellence: II.

Green, C.S. \& Bavelier, D. (2003). "Action video game modifies visual selective attention". Nature, 423, 534-537. https://doi.org/10.1038/nature01647

Hakoda Y, \& Sasaki M. (1990). Group version of the stroop and reverse-stroop test-the effect of reaction mode, order, and practice. Jpn. J. Educ. Psychol., 39, 231-239. https://doi.org/10.5926/ijep1953.38.4 389

Hakoda Y, \& Sasaki M. (1991). Two kinds of interference in group version of the Stroop and ReverseStroop Test: the effects of reaction mode. Kyushyu Uni. Rep. Couns. Guid. Stud., 69-81.

Hotta, M. \& Kohata, Y. (2017). The evaluation of usability of EC site using electroencephalogram (EEG). Proc. 19th Jpn. Soc. Kansei Eng., 1-5.

Kakei, S. (2017). Possibility of e-sports. CUC view \& vision, 43, 16-20.

Konami Holdings Corporation (2018, August 30). Pro Evolution Soccer 2019. Retrieved from https://www.konami.com/wepes/2019/jp/jal

Littlesoftware (2018, November 10). Kansei Module logger. Retrieved from http://www.littlesoftware.jp/product/

Mitsukura, Y. (2016). KANSEl Analyzing by EEG. J. Inst. Electr. Eng. Jpn., 136, 687-690. https://doi.org/10.1541/ieejjournal.136.687

Nideffer, R. M. (1985). Athletes' guide to mental training. Champaign, IL: Human Kinetics.

Ohkubo, T., Tamamaru, K. \& Koshimizu, S. (2018). Development of the Impression Detection System by using a Portable EEG Device for Tourist Impression Analysis. Trans. Jpn. Soc. Kansei Eng., 17, 285291. https://doi.org/10.5057/jiske.TJSKE-D-17-00082

Omori, K. (2017). Effect of an acute low-intensity exercise on Stroop Color-Word Test performance. Suruga Univ. Stud. 54, 1-5. 
Pessoa, L. (2009). How do emotion and motivation direct executive control? Trends Cog. Sci. 13(4), 160 166. https://doi.org/10.1016/.tics.2009.01.006

Shibata, S., Akita, T. \& Kimura, H. (2015). Emotion extraction method for listening to the pleasant and unpleasant sound using alpha and beta wave on a simplified EEG. Bull. Daido Univ., 51, 97-103.

Song, Y. \& Hakoda, Y. (2015). An fMRI study of the functional mechanisms of Stroop/reverse-Stroop effects. Behav. Brain Res., 290, 187-96. https://doi.org/10.1016/i.bbr.2015.04.047

Thoughts and affect occurring during competition. Sport Psychol. 6, 383-402. https://doi.org/10.1123/tsp.6.4.383 